\title{
Nonlinear System Identification Based on Ensemble Learning: The Cascaded Tanks System Case
}

\author{
Matheus Henrique Dal Molin Ribeiro*,** \\ Viviana Cocco Mariani ${ }^{* * *, * * * *}$ Luiz Felipe Manke ${ }^{\dagger}$ \\ Leandro dos Santos Coelho ${ }^{*, * * * *}$ \\ * Industrial and Systems Engineering Graduate Program (PPGEPS), \\ Pontifical Catholic University of Parana (PUCPR) Curitiba, Parana, \\ Brazil, (email:leandro.coelho@pucpr.br) \\ ** Federal Technological University of Parana (UTFPR), Pato Branco, \\ Parana,Brazil (email:mribeiro@utfpr.edu.br) \\ *** Mechanical Engineering Graduate Program (PPGEM), Pontifical \\ Catholic University of Parana (PUCPR) Curitiba, Parana, \\ Brazil,(email:viviana.mariani@pucpr.br) \\ **** Department of Electrical Engineering, Federal University of \\ Parana (UFPR), Curitiba, Parana, Brazil \\ ${ }^{\dagger}$ WEG Electrical Equipment S.A., Department of Research and \\ Technological Innovation, Jaragua do Sul, Santa Catarina, Brazil, \\ (email:luizmanke@gmail.com)
}

\begin{abstract}
Ensemble approach plays an important role in the currently scientific scenario, once it can be used to improve the accuracy of time series models. This approach combines several weak models to build an efficient forecasting model able to deal with nonlinear systems. In this aspect, the aim of this paper is to propose an ensemble approach based on stacking to make predictions $k$-step-ahead for a cascaded tank system benchmark problem. Base learners (BL) such as partial least squares, support vector machines for regression, boosted generalized linear and weighted $k$-nearest neighbors models are adopted as well as boosted linear model is used as meta-learner. The performance of proposed ensemble is compared with BL performance by root mean squared error, determination coefficient and mean absolute error criteria. Results show that performance of proposed approach are better than the BL performance. Indeed, satisfactory forecasting results regarding to accuracy are found.
\end{abstract}

Keywords: Cascaded tank system, ensemble learning, time series, nonlinear system identification, stacked generalization.

\section{INTRODUCTION}

The mathematical modeling of a system is an approach whose objective is to develop an algebraic expression able to describes its behavior. In this context, it is expected that the function found can be applied to monitor the described system and be useful to know the future responses. Hence, in this aspect, it is possible to develop a strategic planning to avoid losses as well as to prevent extreme events. Nonetheless, most of the real systems are characterized as nonlinear, and find an adequate transference function to them is a challenge, because it is necessary to make predictions with assertiveness and they should make sense in the real problem domain.

Considering the above mentioned, the modeling of these situations can be include in nonlinear system identification field. Faced with this, the development of models based on artificial intelligence (AI), mathematical and statistical approaches able to modeling inherent to data dynamic should be explored. In this context, a recent benchmark problem namely cascaded tank system (CTS) was proposed, which goal is to estimate a model able to make accurate predictions of water level in the first reservoir (Schoukens et al., 2016; Schoukens and Noël, 2017).

Due to the importance of modeling of system above mentioned, some studies have already been conducted in this research field. The first approach was proposed to identify an unstructured flexible nonlinear state space model (Relan et al., 2017). Regarding to the root mean squared error (RMSE) criterion the proposed approach shows superior results in relation to two other approaches. In the sequence, the second study proposed a piecewise autoregressive exogenous (PWARX) model (Mattsson et al., 2018). By comparing the RMSE, this study shows that the PWARX model improves the result by switching between different linear models depending on the current water level and inflow into the lower tank, regarding to autoregressive exogenous model. Finally, the CTS was modeled by Volterra series model using regularized basis function in a bayesian framework (Stoddard and Welsh, 2018). As 
results, the authors argued that the proposed approach is more compact that a time domain formulation and could be estimated several orders of magnitude faster and shows better results than other approaches proposed in the literature.

Regarding to AI perspective, and due to the aforementioned data sets organized in the temporal context, approach that can be employed in systems identification is the ensemble (EM), especially, stacked generalization (STACK) (Wolpert, 1992). EM is an methodology used to increase models' accuracy, which combines (by weighted averaging rule in regression problems) the prediction from several base (weak) learners for the same data set. Indeed, faced with this, it is expected obtain an accurate model.

The STACK ensemble is an EM framework that executes training process by layers or levels. In this context, base learners (BL) are trained in the first level (level-0) and its predictions are used as features in the subsequent level. In the next stage, a meta-learner (MLE) is some model able to deal with the characteristics of the original data set. From this model, the final predictions are obtained. Several studies have already used this approach with success in the regression and classification fields as presented in Chen et al. (2014); Sun and Trevor (2018); Shan et al. (2019); Wang et al. (2019).

Considering the previous review, the objective of this paper is to propose an ensemble model based on STACK approach for CTS benchmark problem (BP) to forecasting $k$-step-ahead the water level in the lower reservoir. In this $\mathrm{BP}$, the purpose is to find a model able to predicted the fluid levels. To attend this, in the proposed model, as BL, four models are used, namely: partial least squares (Fuentes et al., 2015), support vector machine with linear kernel (SVR) (Sahoo et al., 2019), boosted generalized linear model (BSTGLM) (Hofner et al., 2014) and weighted k-Nearest Neighbors (WKNN) (Talavera-Llames et al., 2019). Also, as MLE is adopted the boosted linear model (BSTLM) (Fernández-Delgado et al., 2019). These models were chosen because they already presented good results in literature for time series forecasting problems.

With objective of evaluate the performance of proposed model, the STACK performance's is compared with BL. The main contribution of this paper is to propose an ensemble model able to capture the nonlinearities of $\mathrm{BP}$, which combines the expertise of several ML associated to feature engineering process. In addition, to our best knowledge, this is the first time that STACK methodology is employed to solve the CTS problem.

The remainder of this paper is structured as follows. Section 2 presents a brief of BP. Section 3 brings an overview of proposed methodology. Section 4 describes the results. Finally, Section 5 presents the conclusion and future research.

\section{BENCHMARK PROBLEM}

The CTS BP is a fluid level control system consisting of two tanks with free outlets fed by a pump. The input signal controls a water pump that pumps the water from a reservoir into the upper water tank. The water of the upper water tank flows through a small opening into the lower water tank, and finally through of a small opening from the lower water tank back into the reservoir (Schoukens et al., 2016; Schoukens and Noël, 2017). The input of the system is the pump voltage $(\mathrm{Hz})$, the output is the water level (l) of the lower tank. Figure 1 shows this procedure.

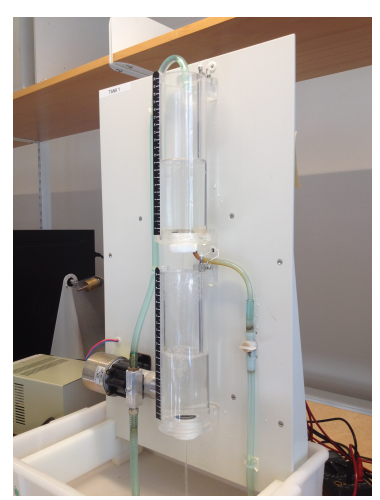

(a)

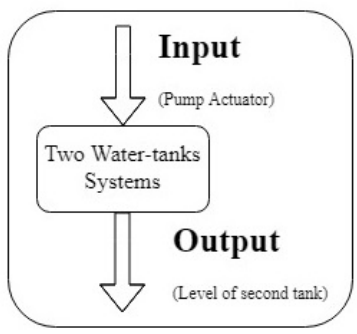

(b)
Figure 1. Representation of CTS Systema (a) and Block Diagram for input and output system (b).

According to Schoukens et al. (2016), the system was not in steady state during the measurements. The systems has an unknown initial value at the start of the measurements, being the same for both the estimation and the test data record.

The mathematical formulation to this problem can be find in Schoukens and Noël (2017). In this system, the input signals $(u(\mathrm{t}))$ are multisine signals which are 1024 observations long and excite the frequency range from 0 to $0.0144 \mathrm{~Hz}$, both for the estimation and test case. The sample period is equal to $4 \mathrm{~s}$, that is, on each $4 \mathrm{~s}$ the water levels is measured. In this case, in the x-axis of Figure 2 , the values exceed 4000s. The input signals are zero order hold. In estimation and validation set there are 1024 observations.

The data set behavior is illustrated in Figure 2.

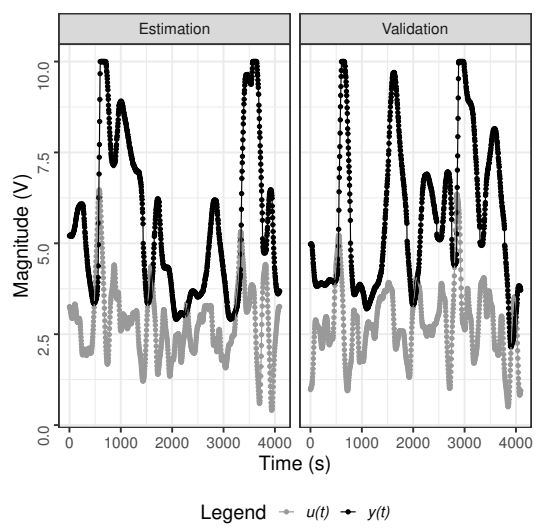

Figure 2. Data sets for models estimation (left) and validation (right) in the time domain.

\section{METHODOLOGY}

This section presents the roadmap used to modeling the CTS problem. In the first step the lags for input (3 
lags) and output (2 lags) are obtained through crosscorrelation and auto-correlation analysis. Second, feature engineering is employed in order to obtain 8 features. The first three are based on lag 1 for input in a time window of three lags, that is, average $(\bar{u})$, standard deviation (SD) and skewness (SKW). The next is based on difference between currently observation for lag 1 of input and early observation. Besides that, the last four are exponential 2 and 3, hyperbolic tangent and logarithm for input lag 1 . Finally, center-scale preprocess is applied to the data sets with the purpose of accommodate variations inherent.

As next step, feature importance (FI) is obtained from boosting approach with purpose of identify and ranking the influences of input variables on output predictions according to expression

$$
\begin{aligned}
\hat{y}(t)= & f\{\hat{y}(t-1), \hat{y}(t-2), u(t-1), \\
& \left.u(t-2), u(t-3), u_{i}(t-1)\right\},
\end{aligned}
$$

in which $\hat{y}(t)$ is the predicted output, $\hat{y}(t-1)$ and $\hat{y}(t-2)$ are the past predicted values for water level, $u(t-j), j=$ $1,2,3$, are the lags for input, and $u_{i}(t-1)$, for $i=1, \ldots, 8$ are the features based on $u(t-1)$ described above. The threshold for FI is set at 0.01. In this aspect, redundant features are removed from data set, once they can reduce the model performance. This approach is developed based on empirical improvement in squared error as a result of using predictor $u_{k}$ as a splitting variable as the nonterminal node. Further details about FI using boosting can be found in Breiman et al. (1984).

As soon as the feature set is defined, the STACK modeling is performed. The expertise adjacent of STACK is to build a meta-training set from the BL predictions based on training sets with purpose of associate them through a learning mechanism to predict the future values. Indeed, BL such as PLS, SVR, BSTGLM and WKNN are trained, in the STACK's layer-0, to predict lower tank's water level according to (1) without the features whose importance is lower than the defined threshold. In this step, time series cross-validation (leave-one-out with time slice) is employed (Bergmeir et al., 2018). In this context, the STACK developed is a heterogeneous, once BL structure as different and diverse. This is a key factor to successful of ensemble learning. In the next phase, once the predictions from BL are obtained, the MLE BSTLM is trained and final predictions are obtained. Figure 3 summarize this process.

With the objective of evaluate the models merit regarding accuracy for validation set, the mean average error (MAE), determination coefficient $\left(R^{2}\right)$ and RMSE criteria are employed, as well as the percentage improvement in the measures, which are stated as follow

$$
\begin{gathered}
M A E=\sum_{i=1}^{n}\left|\frac{y_{i}(t)-\hat{y}_{i}(t)}{n}\right|, \\
R M S E=\sqrt{\frac{1}{n} \sum_{i=1}^{n}\left[y_{i}(t)-\hat{y}_{i}(t)\right]^{2},}
\end{gathered}
$$

$$
\begin{gathered}
R^{2}=1-\frac{\sum_{i=1}^{n}\left[y_{i}(t)-\hat{y}_{i}(t)\right]^{2}}{\sum_{i=1}^{n}\left[y_{i}(t)-\bar{y}_{i}(t)\right]^{2}}, \\
P_{R^{2}}=100 \times \frac{R_{P}^{2}-R_{R}^{2}}{R_{P}^{2}}, \\
P_{M A E}=100 \times \frac{M A E_{P}-M A E_{R}}{M A E_{P}}, \\
P_{R M S E}=100 \times \frac{R M S E_{P}-R M S E_{R}}{R M S E_{P}},
\end{gathered}
$$

in which $y_{i}(t)$ is the output value, $\hat{y}_{i}(t)$ is the predict value by STACK model and $\bar{y}_{i}(t)$ is the average of output values both in instant $t=0,4, \ldots, 4096, n=1024$ and $P$ and $R$ indicate proposed and reference model.

In addition, with purpose of evaluate the improvement of adopted methodology, Friedman and Wilcoxon Signed rank tests are applied. The first test is adopt to check if there is difference between the errors from used algorithms, while the second test is adopted to verify which algorithms show statistically significant difference in errors. Further details can be found in Demšar (2006) and García et al. (2010).

Figure 3 shows the roadmap for CTS modeling.

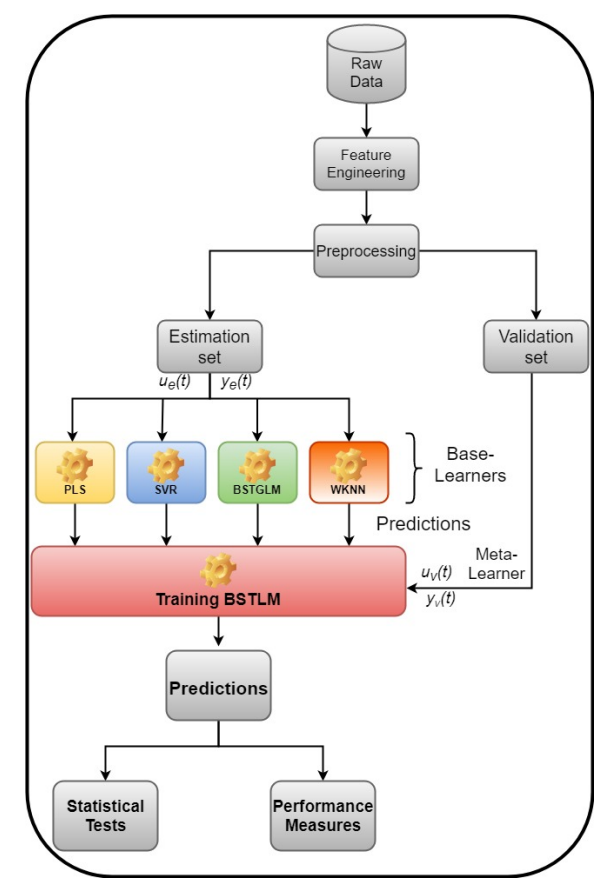

Figure 3. Roadmap for proposed STACK model.

Results described in Section 4 are generated using the processor Intel(R) Core(TM) i5-4200U, 8bg of random access memory and central processing unit with $1.6 \mathrm{~Hz}$ and Windows 10 operating system. The caret (Kuhn, 2008) package available in $\mathrm{R}$ software ( $\mathrm{R}$ Core Team, 2019) is used to obtain the results of this paper. 


\section{RESULTS}

This section report the results for exploratory data analysis as well as the results obtained from STACK model. In this context, Table 1 summarizes the descriptive measures for remain features after performed FI.

Table 1. Descriptive measures and importance for each feature used in STACK model.

\begin{tabular}{c|c|c|c}
\hline Measure & $y(t-1)$ & $y(t-2)$ & $u(t-1)$ \\
\hline Minimun & $-1.28 \mathrm{E}+00$ & $-1.28 \mathrm{E}+00$ & $-2.29 \mathrm{E}+00$ \\
$1^{\text {st }}$ Quantile & $-7.61 \mathrm{E}-01$ & $-7.60 \mathrm{E}-01$ & $-5.97 \mathrm{E}-01$ \\
Median & $-1.71 \mathrm{E}-02$ & $-1.71 \mathrm{E}-02$ & $-7.12 \mathrm{E}-02$ \\
$\bar{x}$ & $-5.56 \mathrm{E}-03$ & $-5.16 \mathrm{E}-03$ & $0.00 \mathrm{E}+00$ \\
$3^{\text {th }}$ Quantile & $4.62 \mathrm{E}-01$ & $4.62 \mathrm{E}-01$ & $6.39 \mathrm{E}-01$ \\
Maximum & $2.37 \mathrm{E}+00$ & $2.37 \mathrm{E}+00$ & $3.55 \mathrm{E}+00$ \\
FI & $1.00 \mathrm{E}+02$ & $2.34 \mathrm{E}+00$ & $3.03 \mathrm{E}-02$ \\
\hline Measure & $u(t-2)$ & $u(t-3)$ & SD \\
\hline Minimun & $-2.30 \mathrm{E}+00$ & $-2.30 \mathrm{E}+00$ & $-1.14 \mathrm{E}+00$ \\
$1^{\text {st }}$ Quartile & $-5.97 \mathrm{E}-01$ & $-5.97 \mathrm{E}-01$ & $-7.58 \mathrm{E}-01$ \\
Median & $-7.13 \mathrm{E}-02$ & $-7.13 \mathrm{E}-02$ & $-3.43 \mathrm{E}-01$ \\
$\bar{x}$ & $0.00 \mathrm{E}+00$ & $0.00 \mathrm{E}+00$ & $0.00 \mathrm{E}+00$ \\
$3^{\text {th }}$ Quartile & $6.39 \mathrm{E}-01$ & $6.39 \mathrm{E}-01$ & $5.48 \mathrm{E}-01$ \\
Maximum & $3.55 \mathrm{E}+00$ & $3.55 \mathrm{E}+00$ & $3.95 \mathrm{E}+00$ \\
FI & $1.74 \mathrm{E}-02$ & $9.16 \mathrm{E}-02$ & $9.77 \mathrm{E}-03$ \\
\hline Measure & SKW & Difference & Exponential 2 \\
\hline Minimum & $-2.74 \mathrm{E}+00$ & $-3.33 \mathrm{E}+00$ & $-1.34 \mathrm{E}+00$ \\
$1^{\text {st }}$ Quartile & $-4.06 \mathrm{E}-01$ & $-5.49 \mathrm{E}-01$ & $-6.24 \mathrm{E}-01$ \\
Median & $0.00 \mathrm{E}+00$ & $-7.57 \mathrm{E}-03$ & $-2.18 \mathrm{E}-01$ \\
$\bar{x}$ & $0.00 \mathrm{E}+00$ & $0.00 \mathrm{E}+00$ & $0.00 \mathrm{E}+00$ \\
$3^{\text {th }}$ Quartile & $4.19 \mathrm{E}-01$ & $5.10 \mathrm{E}-01$ & $4.67 \mathrm{E}-01$ \\
Maximum & $2.82 \mathrm{E}+00$ & $3.36 \mathrm{E}+00$ & $4.93 \mathrm{E}+00$ \\
FI & $5.67 \mathrm{E}-03$ & $1.87 \mathrm{E}-02$ & $4.08 \mathrm{E}-03$ \\
\hline
\end{tabular}

Table 2 presents control hyperparameters for BL models after perform cross-validation process. In this paper, only seven parameters are defined.

Table 2. Control hyperparameters for BL and proposed approach.

\begin{tabular}{c|c|c}
\hline Model & \multicolumn{2}{|c}{ Control Hyperparameters } \\
\hline STACK & Number of trees & 150 \\
& Shrinkage & 0.1 \\
\hline BSTGLM & Number of trees/Prune & $100 /$ No \\
\hline SVR & Cost/Kernel & $1 /$ Linear \\
\hline PLS & Number of components & 3 \\
\hline WKNN & Max. Number of Neighbors & 9 \\
& Distance & 2 \\
\hline
\end{tabular}

Table 3 presents the performance measures employed for models evaluation.

Table 3. Performance measures for models evaluation.

\begin{tabular}{c|c|c|c}
\hline & \multicolumn{3}{|c}{ Estimation set } \\
\hline Models & RMSE & $R^{2}$ & MAE \\
\hline STACK & 0.0280 & 0.9992 & 0.0177 \\
BSTGLM & 0.0293 & 0.9991 & 0.0181 \\
PLS & 0.0409 & 0.9983 & 0.0257 \\
SVR & 0.0407 & 0.9987 & 0.0309 \\
WKNN & 0.0280 & 0.9992 & 0.0138 \\
\hline & \multicolumn{3}{|c}{ Validation set } \\
\hline STACK & 0.3667 & 0.8792 & 0.2965 \\
BSTGLM & 0.3823 & 0.8667 & 0.3072 \\
PLS & 0.3857 & 0.8573 & 0.3107 \\
SVR & 0.6247 & 0.7196 & 0.4808 \\
WKNN & 0.7624 & 0.4741 & 0.5429 \\
\hline
\end{tabular}

It is possible to identify through of results of Table 3 the STACK as the best modeling. For the estimation set, the STACK's results are better than three BL and similar to WKNN model. In the validation set, regarding of RMSE and MAE analysis, this approach reduce these criteria ranged between $4.08 \%-51.90 \%$ and $3.48 \%-45.39 \%$. In addition, with regard to $R^{2}$, the meta-modeling increase this measure ranged between $1.42 \%$ and $46.08 \%$. Hence, it is able to make prediction with lower errors and can explain a higher percentage of the data variance.

The superiority of the STACK proposed in this paper corroborates with a range of results from other areas (Anifowose et al., 2015; Chu et al., 2019; Shan et al., 2019). This approach is able to deal with intrinsic characteristics of the data such as nonlinearity and this is coherent with the STACK's specialty. Besides that, this model needs only 7 parameters to be defined and it is an advantage. This make the process faster, once the time process is $0.4 \mathrm{~min}$. Although the approaches proposed by Pan and Welsh (2018) and Mattsson et al. (2018) showed good results regarding accuracy, they need more parameters to be adjusted ( $\approx 8$ and 585 respectively) than the proposed system in this paper, and it is a disadvantage, once the results (RMSE) obtained are slightly similar those presented in this paper ( 0.3617 and 0.35 respectively). In these cases, the computational time to estimate the model is high depending on the computational settings.

By Friedman test, there is difference between squared errors (SE) from different models $\left(\chi_{4}^{2}=470.66, p\right.$-value $<0.01)$. Table 4 shows the statistic for Wilcoxon signed rank test (two tail) and $p$-values to compare the SE of BL and STACK models.

Table 4. Results from Wilcoxon signed rank test.

\begin{tabular}{c|c|c}
\hline Model & $\chi^{2}$ & $p$-value \\
\hline PLS & $6.10 \mathrm{E} 5$ & $<0.01$ \\
SVR & $4.01 \mathrm{E} 5$ & $<0.01$ \\
BSTGLM & $5.94 \mathrm{E} 5$ & $<0.01$ \\
WKNN & $4.38 \mathrm{E} 5$ & $<0.01$ \\
\hline
\end{tabular}

Figure 4 shows the STACK predictions for $k$-step-ahead on validation set.

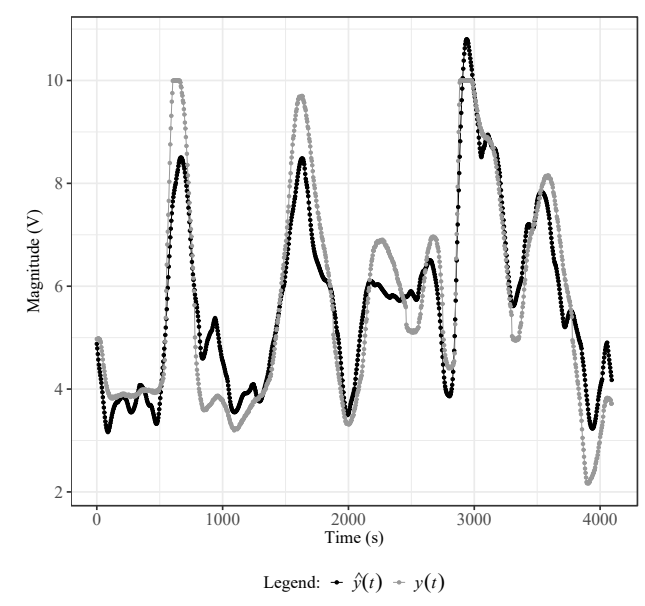

Figure 4. Predicted and observed values for water level in the time domain from STACK model. 
It can be verified that predictions from proposed approach are close to real values. In the ups and downs the model was not able to capture the extreme behavior. This is associated to recursive predictions $k$-step-ahead developed in STACK model, which accumulates the errors of the previous predictions for the next. However, in general lines the model learned partially the data dynamic, even in the presence of different ways of nonlinearities.

In addition, Figure 5 illustrated the forecasting values from PLS (A), SVR (B), BSTGLM (C) and WKNN (D).
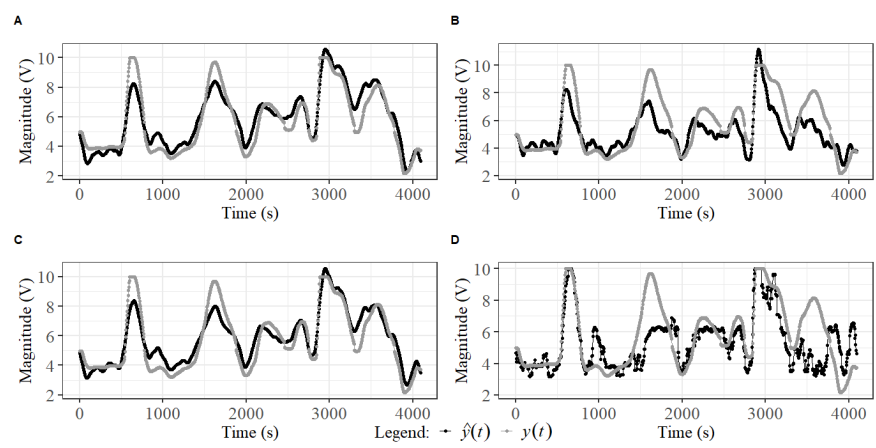

Figure 5. Predicted and observed values for water level in the time domain from BL.

Figures 6 and 7 can be employed to check the residuals behavior for estimation set. The first refers to histogram for the errors and second is the simulated envelope for the randomized quantile residuals. In both cases it is suggested that the residuals are white noises. In this perspective, this information in supported by Kolmogorov-Smirnov test (D $=0.0333, p$-value $>0.01$ )

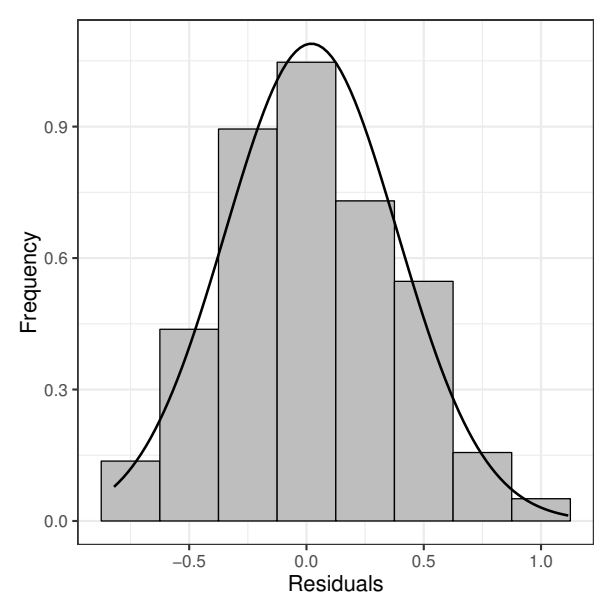

Figure 6. Residuals' frequency distribution.

\section{CONCLUSION AND FUTURE RESEARCH}

This paper proposed an ensemble approach based on STACK with objective of forecasting $k$-step-ahead the water level in a reservoir for the CTS BP. The STACK's level0 was composed by four BL namely: PLS, SVR, BSTGLM and WKNN. In the follow step, the BSTLM model was employed as MLE. The control hyperparameters for both BL and MLE were obtained in training (estimation) phase by grid search during cross-validation process.

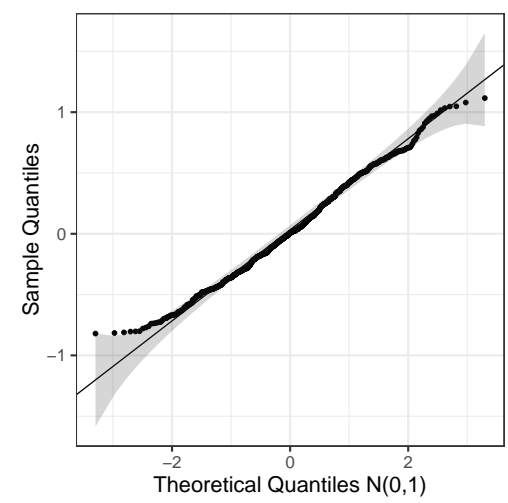

Figure 7. Simulated envelope for the randomized quantile residuals.

In this context, good results regarding accuracy were obtained from proposed approach. The STACK modeling outperform the BL, once lower RMSE - MAE and higher $R^{2}$ were observed. In addition, statistical tests showed that this approach was able to make predictions with lower SE. Even that STACK modeling presented satisfactory results, improvements can be made. The main gain from this model regarding the models pointed in the literature is that this approach needs only 7 parameters to be adjusted, while most of them need more than this. This makes the process fast.

As future research is intend to: adopting other BL as well as MLE, make signal decomposition using ensemble empirical mode decomposition, adjust control hyperparameters through single and multi-objective optimization.

\section{ACKNOWLEDGMENT}

The authors would like to thank National Council of Scientific and Technologic Development of Brazil - CNPq (Grants: 150501/2017-0-PDJ, 303906/2015-4-PQ, 303908/ 2015-7-PQ, 405101/2016-3-Univ, 404659/2016-0-Univ) and Fundacao Araucaria (PRONEX-FA/CNPq 042/2018) for its financial support of this work. Furthermore, the authors wish to thank the Editor and anonymous referees for their constructive comments and recommendations, which have significantly improved the presentation of this paper.

\section{REFERENCES}

Anifowose, F., Labadin, J., and Abdulraheem, A. (2015). Improving the prediction of petroleum reservoir characterization with a stacked generalization ensemble model of support vector machines. Applied Soft Computing, 26, 483-496. doi:10.1016/j.asoc.2014.10.017.

Bergmeir, C., Hyndman, R.J., and Koo, B. (2018). A note on the validity of cross-validation for evaluating autorregressive time series prediction. Computational Statistics \& Data Analysis, 120, 70-83. doi:10.1016/j. csda.2017.11.003.

Breiman, L., Friedman, J., Stone, C., and Olshen, R. (1984). Classification and Regression Trees. The Wadsworth and Brooks-Cole statistics-probability series. Taylor \& Francis.

Chen, Y., Wong, M.L., and Li, H. (2014). Applying ant colony optimization to configuring stacking ensembles 
for data mining. Expert Systems with Applications, 41(6), 2688 - 2702. doi:10.1016/j.eswa.2013.10.063.

Chu, J., Yuan, L., Pan, L., Liu, Q., Yan, J., and Liu, Y. (2019). Nwp combination correction model based on variable-weight stacking algorithm. Energy Procedia, 158, 6309-6314. doi:10.1016/j.egypro.2019.01.408.

Demšar, J. (2006). Statistical comparisons of classifiers over multiple data sets. J. Mach. Learn. Res., 7, 1-30. URL http://dl.acm.org/citation.cfm?id= 1248547.1248548.

Fernández-Delgado, M., Sirsat, M., Cernadas, E., Alawadi, S., Barro, S., and Febrero-Bande, M. (2019). An extensive experimental survey of regression methods. Neural Networks, 111, 11-34. doi:10.1016/j.neunet.2018.12.010.

Fuentes, J., Poncela, P., and Rodriguez, J. (2015). Sparse partial least squares in time series for macroeconomic forecasting. Journal of Applied Econometrics, 30, 576594. doi:10.1002/jae.2384.

García, S., Fernández, A., Luengo, J., and Herrera, F. (2010). Advanced nonparametric tests for multiple comparisons in the design of experiments in computational intelligence and data mining: Experimental analysis of power. Information Sciences, 180(10), 2044 - 2064. doi: 10.1016/j.ins.2009.12.010. Special Issue on Intelligent Distributed Information Systems.

Hofner, B., Mayr, A., Robinzonov, N., and Schmid, M. (2014). Model-based boosting in R: a hands-on tutorial using the $\mathrm{R}$ package mboost. Computational Statistics, 29(1), 3-35. doi:10.1007/s00180-012-0382-5.

Kuhn, M. (2008). Building predictive models in R using the caret package. Journal of Statistical Software, Articles, 28(5), 1-26. doi:10.18637/jss.v028.i05.

Mattsson, P., Zachariah, D., and Stoica, P. (2018). Identification of cascade water tanks using a PWARX model. Mechanical Systems and Signal Processing, 106, 40-48. doi:10.1016/j.ymssp.2017.12.027.

Pan, S. and Welsh, J.S. (2018). An application of indirect inference to the cascaded tanks nonlinear benchmark. IFAC-PapersOnLine, 51(15), 790-795. doi:10.1016/j. ifacol.2018.09.134. 18th IFAC Symposium on System Identification SYSID 2018.

R Core Team (2019). R: A Language and Environment for Statistical Computing. R Foundation for Statistical Computing, Vienna, Austria. URL http://www . R-project.org/.

Relan, R., Tiels, K., Marconato, A., and Schoukens, J. (2017). An unstructured flexible nonlinear model for the cascaded water-tanks benchmark. IFAC-PapersOnLine, 50(1), 452-457. doi:10.1016/j.ifacol.2017.08.074.

Sahoo, B.B., Jha, R., Singh, A., and Kumar, D. (2019). Application of support vector regression for modeling low flow time series. KSCE Journal of Civil Engineering, 23(2), 923-934. doi:10.1007/s12205-018-0128-1.

Schoukens, M., Mattson, P., Wigren, T., and Noël, J. (2016). Cascaded tanks benchmark combining soft and hard nonlinearities. In Workshop on Nonlinear System Identification Benchmarks, 20-23. Brussels, Belgium.

Schoukens, M. and Noël, J. (2017). Three benchmarks addressing open challenges in nonlinear system identification. IFAC-PapersOnLine, 50(1), 446-451. doi: 10.1016/j.ifacol.2017.08.071.

Shan, P., Zhao, Y., Wang, Q., Sha, X., Lv, X., Peng, S., and Ying, Y. (2019). Stacked ensemble extreme learning machine coupled with partial least squaresbased weighting strategy for nonlinear multivariate calibration. Spectrochimica Acta Part A: Molecular and Biomolecular Spectroscopy, 215, 97-111. doi:10.1016/j. saa.2019.02.089.

Stoddard, J.G. and Welsh, J.S. (2018). Regularized basis function estimation of volterra kernels for the cascaded tanks benchmark. IFAC-PapersOnLine, 51(15), 413418. doi:10.1016/j.ifacol.2018.09.180.

Sun, W. and Trevor, B. (2018). A stacking ensemble learning framework for annual river ice breakup dates. Journal of Hydrology, 561, 636-650. doi:10.1016/j. jhydrol.2018.04.008.

Talavera-Llames, R., Pérez-Chacón, R., Troncoso, A., and Martínez-Álvarez, F. (2019). MV-KWNN: A novel multivariate and multi-output weighted nearest neighbours algorithm for big data time series forecasting. Neurocomputing (in press). doi:10.1016/j.neucom.2018.07. 092.

Wang, Y., Wang, D., Geng, N., Wang, Y., Yin, Y., and Jin, Y. (2019). Stacking-based ensemble learning of decision trees for interpretable prostate cancer detection. Applied Soft Computing, 77, 188-204. doi:10.1016/j.asoc.2019. 01.015 .

Wolpert, D.H. (1992). Stacked generalization. Neural Networks, 5(2), 241 - 259. doi:10.1016/S0893-6080(05) 80023-1. 